\title{
Paraneoplastische Dermatomyositis bei papillärem Ovarialkarzinom
}

\author{
Paraneoplastic Dermatomyositis in a Patient Suffering from Ovarial Carcinoma
}

Autoren

Institut

\section{Pfütze, O. Petzold, H. Löffler}

Hautklinik der SLK-Kliniken Heilbronn, Akademisches Lehrkrankenhaus der Universität Heidelberg

\section{Bibliografie}

DOI $10.1055 / \mathrm{s}-2008-1077604$

Akt Dermatol 2008; 34:

376-379 @ Georg Thieme

Verlag KG Stuttgart · New York ISSN 0340-2541

Korrespondenzadresse

Prof. Dr. Harald Löffler

Hautklinik

SLK-Kliniken

Am Gesundbrunnen 20-24

74078 Heilbronn

Harald.Loeffler@slk-kliniken.de

\section{Zusammenfassung \\ $\nabla$}

Die Dermatomyositis ist eine Autoimmundermatose ungeklärter Ätiologie, die in ca. 25 \% der Fälle als Paraneoplasie auftritt. Im vorliegenden Fall war bei einer 68-jährigen Patientin ein Jahr nach Diagnose eines Ovarialkarzinoms eine Dermatomyositis aufgetreten. Unter der daraufhin erfolgten Therapie mit Prednisolon und der Einleitung einer Chemotherapie des Ovarialkarzinoms war primär eine gute Krankheitskontrolle zu erreichen. Im weiteren Verlauf führten kutane Erosionen der Dermatomyositis, welche jeweils als Nebenwirkung der Zytostatika gedeutet wurden,

\section{Einleitung}

$\nabla$

Die Dermatomyositis ist mit einer Inzidenz von 5-10/1000 000 eine relativ seltene Autoimmundermatose. Sie führt vor allem zu Entzündungen der Haut und der Skelettmuskulatur, tritt jedoch gelegentlich auch ohne Myositis auf. Abgesehen von der selteneren juvenilen Dermatomyositis liegt der Altersgipfel zwischen 40 und 60 Jahren. In bis zu $25 \%$ der Fälle handelt es sich um eine paraneoplastische Erkrankung.

\section{Kasuistik \\ V}

\section{Anamnese}

Die 68-jährige Patientin stellte sich mit einem seit fünf Monaten zunehmenden Erythem des Gesichtes und der oberen Extremitäten vor. Daneben habe sich im gleichen Zeitraum vor allem im Bereich der Arme eine progrediente Kraftminderung entwickelt, die von einem generalisierten Leistungsknick begleitet worden war. Innerhalb der letzten 8 Monate sei ein Gewichtsverlust von $18 \mathrm{~kg}$ aufgefallen. Des Weiteren war bei der Patientin ein serös papilläres Ovarialkarzinom wiederholt zum Umsetzen bzw. Absetzen der Chemotherapie. Parallel fand sich eine Progression des Ovarialkarzinoms. Unter der Wiederaufnahme der Therapie zeigte sich die Dermatomyositis erneut regredient. Dieser Fall zeigt, dass durch interdisziplinäre Zusammenarbeit zwischen Onkologen und Dermatologen eine verbesserte Therapiekontrolle der paraneoplastischen Dermatomyositis erreicht werden kann. Zudem unterstützt der vorliegende Fall die Hypothese, dass die Krankheitsaktivität der paraneoplastischen Dermatomyositis eng mit der Tumoraktivität verknüpft ist und eine onkologische Therapie zur Regredienz der Dermatomyositis beiträgt.

(pT3c Nx Mx, G2) mit Z.n. Adnektomie und Omentektomie (R2-Resektion) ein Jahr zuvor bekannt, aufgrund dessen eine anschließende Chemotherapie mit sechs Zyklen Carboplatin/Taxol durchgeführt worden war. Weiterhin bestand seit zwei Jahren eine seronegative chronische Polyarthritis der Hände und Unterarme, welche bis zum Zeitpunkt der Aufnahme mit Methotrexat $10 \mathrm{mg} /$ Woche p.o. und Prednisolon 2,5 mg/d behandelt wurde.

\section{Klinischer Aufnahmebefund}

Bei initialer Vorstellung zeigte sich eine Patientin in deutlich reduziertem Allgemeinzustand. Unter maximaler Anstrengung war im Humeroscapulargelenk eine Elevation bis maximal $90^{\circ}$ gegen die Schwerkraft möglich. An den Unterarmen und Oberschenkeln sowie am Dekolletee und im Gesicht zeigten sich livide bzw. heliotrope, kleinfleckige, teils konfluierende Erytheme und vereinzelte Papeln. An den Fingerstreckseiten fielen gleichartige Erytheme und Gottron'sche Papeln auf. Zudem fanden sich an den distalen Phalangen Nagelfalzhyperkeratosen und vereinzelte Rhagaden ( $\bullet$ Abb. 1). Daneben fiel ein hypomimischer bis depressiver Gesichtsausdruck auf. 

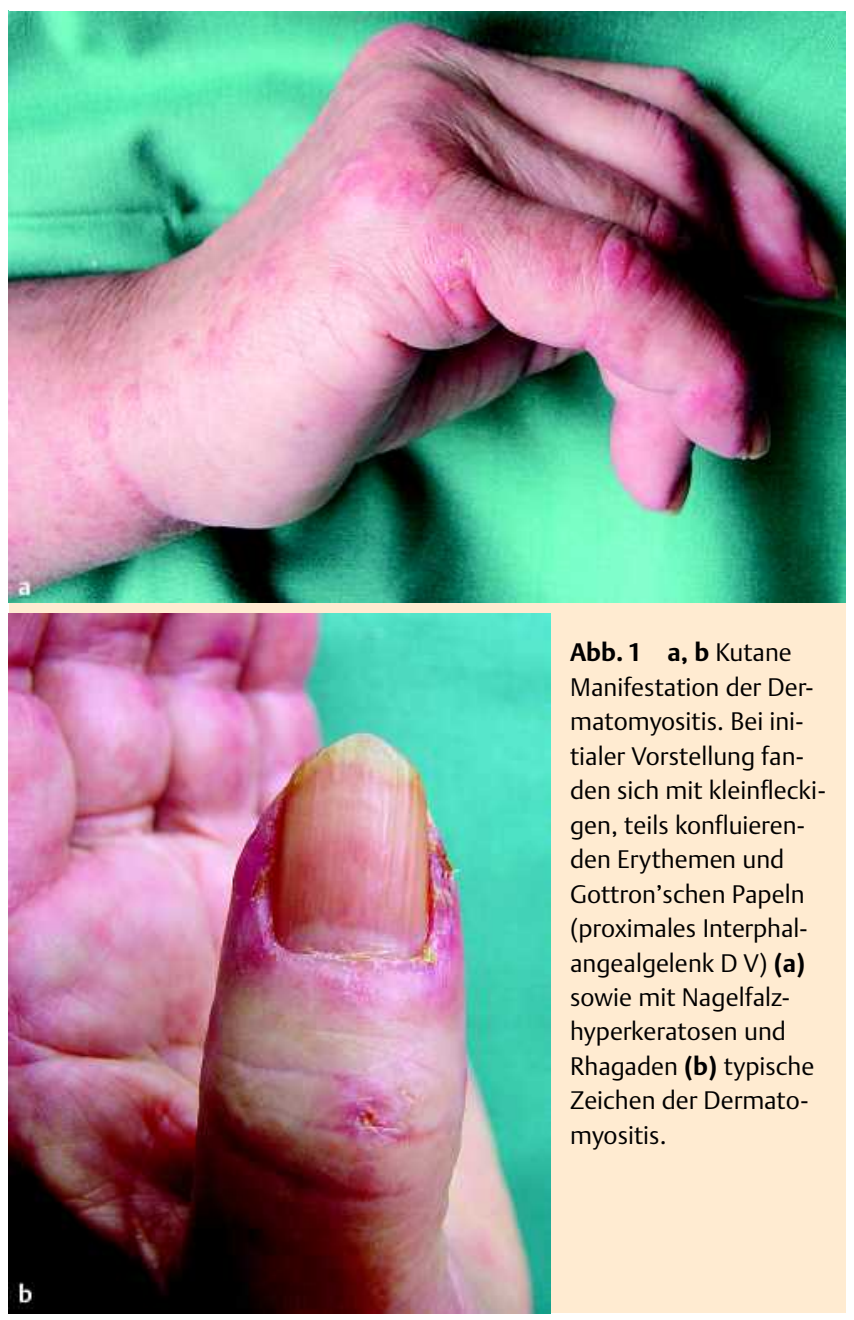

Abb. 1 a, b Kutane Manifestation der Dermatomyositis. Bei initialer Vorstellung fanden sich mit kleinfleckigen, teils konfluierenden Erythemen und Gottron'schen Papeln (proximales Interphalangealgelenk D V) (a) sowie mit Nagelfalzhyperkeratosen und Rhagaden (b) typische Zeichen der Dermatomyositis.

\section{Laborbefunde}

BSG 18/32 mm n.W., GOT 39 U/l, GGT 43 U/l, LDH 291 U/l, CK gesamt $308 \mathrm{U} / \mathrm{l}$ (Normwert: < $145 \mathrm{U} / \mathrm{l}$ ).

Die übrigen Laborparameter der klinischen Chemie und des Differenzialblutbildes sowie die Aldolase waren unauffällig.

Urinstatus: Urin-Kreatinin $38 \mathrm{mg} / \mathrm{dl}$, Urin-Kreatinin $652 \mathrm{mg} /$ $24 \mathrm{~h}$, Kreatinin-Clearance $75 \mathrm{ml} / \mathrm{min}$.

Autoantikörper: ANA negativ, anti-Mi2- und anti-Jo1-Antikörper negativ.

\section{Weitere Befunde}

In der histopathologischen Begutachtung einer Papel fand sich ein eher atrophisches Epithel mit vakuolärer Degeneration der Basalzellschicht und diskreten Muzinablagerungen. Zeichen einer lichenoiden Interface Dermatitis.

Sonografie: Links in Adnexposition teils solide, teils zystische, $30 \times 19 \mathrm{~mm}$ große Raumforderung sowie kranial hiervon eine $46 \times 24 \mathrm{~mm}$ große zystische Raumforderung.

CT-Abdomen: $42 \times 35$ mm große, zystische, teils septierte Raumforderung in Adnexposition links mit medialseitiger Ausziehung; $1 \mathrm{~cm}$ großer LK rechts iliacal. Sonst kein Nachweis suspekter Lymphknotenvergrößerungen.

Röntgen Thorax, Herzechografie und Muskelbiopsie des M. deltoideus unauffällig.

\section{Klinischer Verlauf \\ $\nabla$}

Es erfolgte eine Therapie mit Prednisolon, initial $100 \mathrm{mg} / \mathrm{d}$ p.o. und anschließend befundadaptierter Dosisreduktion bis auf $60 \mathrm{mg} / \mathrm{d}$. Die Therapie der seronegativen chronischen Polyarthritis mit Methotrexat wurde beendet. Im Verlauf von 9 Tagen kam es hierunter zu einer deutlichen Verbesserung des Allgemeinzustandes sowie zum Absinken der Kreatinkinase auf einen Normwert von $70 \mathrm{U} / \mathrm{l}$. Eine Elevation der Arme über $110^{\circ}$ war möglich. Eine erneute operative Therapie des R2-resezierten Ovarialkarzinoms wurde von den Kollegen der Gynäkologie aufgrund der vorbeschriebenen multiplen peritonealen Verwachsungen und der hierdurch bedingt ausgeprägten Radikalität eines möglichen Eingriffes eher ablehnend beurteilt. Im Rahmen der interdisziplinären Tumorkonferenz wurde daher die Einleitung einer Chemotherapie mit liposomalem Doxorubicin vereinbart.

Während des ersten Zyklus der Therapie bildeten sich im Bereich der distalen Phalangen beidseits multiple Nekrosen aus, welche durch die behandelnden Hämato-Onkologen als kutane Nebenwirkung der Chemotherapie gewerteten wurden. Es erfolgte daher eine Umstellung auf das immunsuppressive Chemotherapeutikum Cyclophosphamid in einer Dosierung von $0,5 \mathrm{~g} / \mathrm{m}^{2}$ Körperoberfläche als Bolus alle drei Wochen. Aufgrund der Fingerkuppennekrosen wurde begleitend eine Therapie mit Iloprost $40 \mu \mathrm{g}$ i.v. über 5 Tage durchgeführt. Unter o.g. Therapie kam es innerhalb eines Monats zu einer vollständigen Abheilung. Im Verlauf konnte die Komedikation mit Prednisolon unter Erscheinungsfreiheit bis auf $10 \mathrm{mg} / \mathrm{d}$ reduziert werden.

Vier Monate später erfolgte die erneute Vorstellung der Patientin in unserer Abteilung mit großflächigen, exsudativen Erosionen im Bereich des gesamten Schultergürtels. Bei fehlenden klinischen oder laborchemischen Zeichen einer Myositis war auf die Weiterführung der Therapie mit Cyclophosphamid wegen des Verdachts auf eine erneute kutane Nebenwirkung vorerst verzichtet worden. Trotz Erhöhung der Prednisolon-Dosierung auf $100 \mathrm{mg} / \mathrm{d}$ trat rasch eine progrediente Verschlechterung der entzündlichen Erosionen auf. Laborchemisch stellten sich Kreatinkinase, Transaminasen und Laktatdehydrogenase zu diesem Zeitpunkt normwertig dar. Wir werteten den klinischen Befund als akute Exazerbation der Dermatomyositis. Interessanterweise zeigte sich auch das Ovarialkarzinom im daraufhin erfolgten Staging erneut progredient.

Wir behielten die Dosierung von Prednisolon kurzfristig bei $100 \mathrm{mg} / \mathrm{d}$, behandelten lokal antiseptisch mit Clioquinol und empfahlen eine weitere Cyclophosphamid-Behandlung. Erst unter der Fortsetzung dieser Therapie (Cyclophosphamid 0,5 g/m² Körperoberfläche) kam es zu einer deutlichen Regredienz ( Abb. 2) und schließlich zur vollständigen Abheilung der Erosionen. Prednisolon konnte unter fortgeführter Cyclophosphamidtherapie bei stabilem klinischem Befund auf $20 \mathrm{mg} / \mathrm{d}$ reduziert werden.

\section{Diskussion}

$\nabla$

Die Dermatomyositis ist eine Autoimmundermatose bisher ungeklärter Ätiologie. Sie imponiert charakteristischerweise durch v.a. periorbital und am Dekolletee sowie an den Ellenbogen und Knien flächig oder fleckig auftretenden, rötlichen bis violetten (heliotropen) Erythemen, die sich zum Teil ödematös darstellen können. Seltener treten Hämorrhagien auf. Gelegentlich finden sich in den gleichen Lokalisationen lichenoide (Gottron'sche) Pa- 


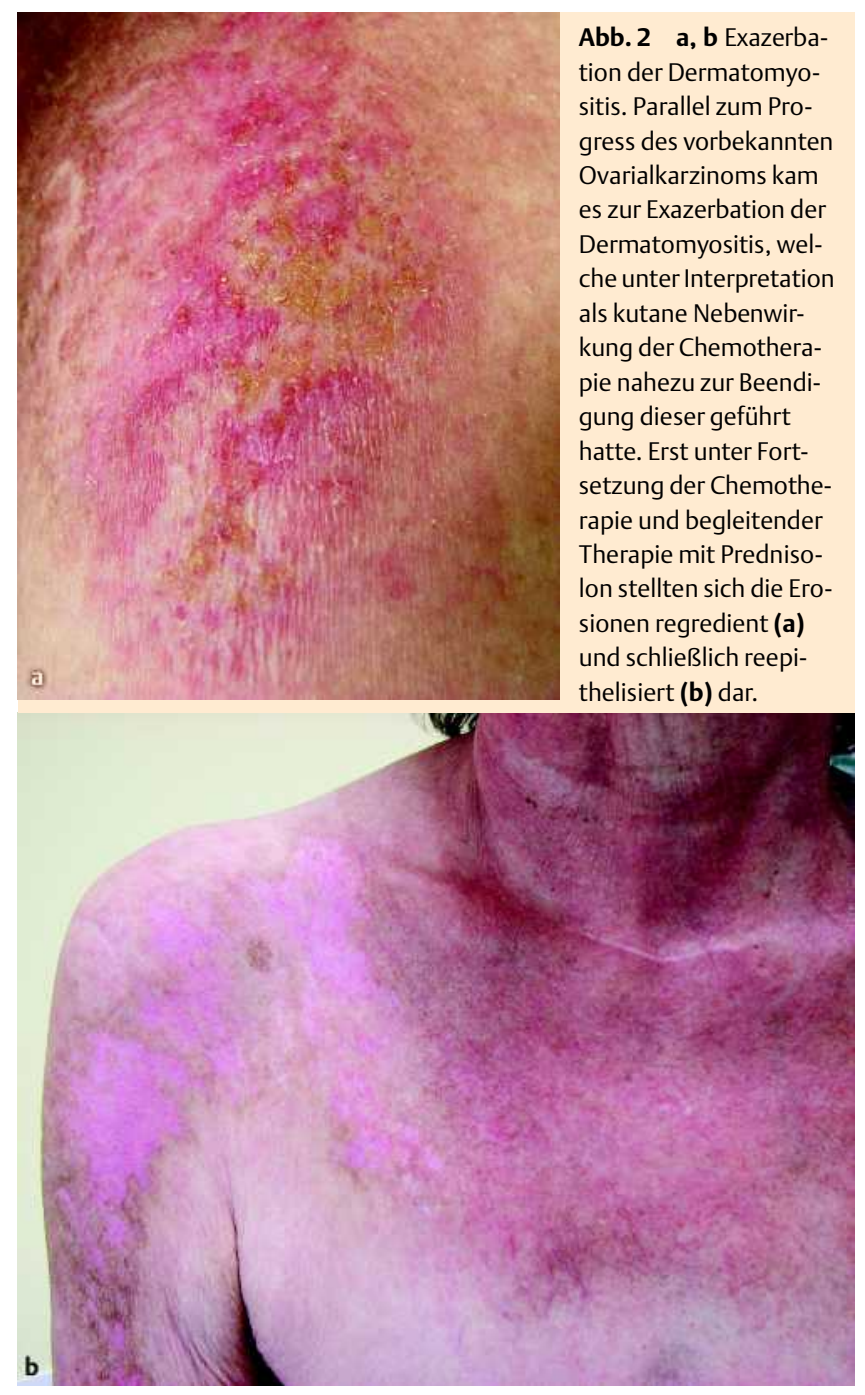

peln. Zudem fallen gehäuft Nagelfalzhyperkeratosen und -hämorrhagien auf. Die Myositis manifestiert sich vor allem im Bereich der proximalen Extremitätenmuskeln. Daneben zeigt die Myositis bei einigen Patienten eine pulmonale, cardiale oder ösophageale Beteiligung.

Pathogenetisch nimmt man an, dass Infektionen mit Picornaund Coxsackie-Viren eine Rolle spielen, da es möglicherweise zur Antikörperbildung gegen Virusoberflächenantigene kommt, welche eine strukturelle Ähnlichkeit mit nukleären Antigenen besitzen [1].

In größer angelegten Kohortenstudien handelte es sich bei 20-25\% der Erkrankungsfälle um eine paraneoplastische Erkrankung [2,3]. Ursächlich hierfür vermutet man im Gegensatz zur oben dargestellten Pathogenese eine Strukturähnlichkeit tumorassoziierter und muskulärer Oberflächenantigene [4]. Bei der Diagnose einer Dermatomyositis sollte daher stets eine entsprechende Tumorsuche durchgeführt werden [5]. Hierbei ist zu beachten, dass die Entität der Tumorerkrankung in der Regel dem alters- und geschlechtsspezifisch zu erwartenden Neoplasierisiko entspricht: Während sich bei Männern gehäuft Kolonund Bronchialkarzinome finden, stellen Mamma- und Ovarialkarzinome die am häufigsten assoziierten Neoplasien bei Frauen dar [3]. Daneben wurde auch gehäuft eine Assoziation zu Bronchial-, Pankreas- und Magenkarzinomen sowie zu NonHodgkin-Lymphomen beschrieben [2].
Die paraneoplastische Dermatomyositis kann jedoch einer klinisch erfassbaren Tumormanifestation vorausgehen. Findet sich zum Zeitpunkt der Diagnosestellung keine assoziierte Neoplasie, sollte daher eine entsprechende Tumorsuche über mindestens drei Jahre jährlich wiederholt werden. Auch danach ist eine an den jeweiligen Fall risikoadaptierte Krebsvorsorge wahrzunehmen [2].

Bei der hier beschriebenen Patientin stellten wir unter Zusammenschau der o.g. Befunde und aufgrund des vorbekannten Ovarialkarzinoms die Diagnose einer paraneoplastischen Dermatomyositis. Entsprechend aktueller Therapieempfehlungen leiteten wir eine systemische Therapie mit oralen Glukokortikoiden in einer Dosierung um $1 \mathrm{mg}$ Prednisolonäquivalent pro kg Körpergewicht ein [6]. Da die Dermatomyositis zumeist eine längerfristige Therapie über mehrere Monate erfordert, wird insbesondere bei therapierefraktären oder schwerwiegenden Fällen zudem eine adjuvante Therapie mit Immunsuppressiva wie Methotrexat, Azathioprin, Cyclophosphamid, Mycophenolat mofetil oder Ciclosporin zur Reduktion glukokortikoidbedingter Nebenwirkungen empfohlen [3,7]. Diese immunsuppressive Therapie stellt jedoch ein Risiko für die Entwicklung von Malignomen sowie (wie im konkreten Fall) für eine mögliche Tumorprogression dar [8,9]. Dagegen wurde wiederholt über vollständige Remission einer Dermatomyositis nach erfolgreicher onkologischer Therapie berichtet [10-13]. Auch unter palliativer Chemotherapie wurde die Remission der paraneoplastischen Dermatomyositis beschrieben [14]. Im vorliegenden Fall verzichteten wir daher auf den Einsatz weiterer Immunsuppressiva und sahen neben der symptomatischen Therapie mit Prednisolon die adäquate Kontrolle des zugrunde liegenden Ovarialkarzinoms als primäres Therapieziel.

Ob die hierunter während des ersten Zyklus mit Doxorubicin aufgetretenen Fingerkuppennekrosen als Nebenwirkung der Chemotherapie oder als weitere Manifestation der Dermatomyositis zu werten sind, ist retrospektiv nicht sicher zu beurteilen. Sie zeigten jedoch primär ein gutes Ansprechen auf den Wechsel der Therapie auf Cyclophosphamid und Iloprost.

Im vorliegenden Fall zeigte sich die Progression der Tumorerkrankung einhergehend mit einer massiven Verschlechterung der Dermatomyositis, welche durch hohe Prednisolongaben schlecht beeinflussbar war. Erst unter der Wiederaufnahme der zytostatischen Therapie mit Cyclophosphamid konnte eine weitgehende Remission der Dermatomyositis erreicht werden. Hier zeigt sich, dass bei einer paraneoplastischen Dermatomyositis nicht die Chemotherapie aufgrund der schweren kutanen Beeinträchtigung eingestellt werden darf (dieses wird von einigen Onkologen gerne gefordert), sondern dass die Behandlung des Tumorleidens essenziell für die Beeinflussung der Dermatomyositis sein kann. Bei Durchführung der Therapie ist eine enge Kooperation der behandelnden Ärzte anzustreben, um kutane Nebenwirkungen der eingesetzten Chemotherapeutika und kutane Manifestationen der Dermatomyositis sicher voneinander abzugrenzen. Auch ist hierbei zu beachten, dass sich die Erkrankung nicht selten als Dermatomyositis sine Myositis darstellen kann. In der Vergangenheit wurde kontrovers diskutiert, ob die Therapie einer Neoplasie zur Abheilung einer paraneoplastischen Dermatomyositis führen kann [3]. Der aktuelle Fall unterstützt die Beobachtung, dass ein entsprechender Zusammenhang besteht und somit eine stadiengerechte onkologische Therapie gleichzeitig essenziell für die Behandlung der paraneoplastischen Dermatomyositis ist. 


\section{Abstract}

\section{Paraneoplastic Dermatomyositis in a Patient Suffering from Ovarial Carcinoma \\ $\nabla$}

Dermatomyositis is an autoimmune skin disease of unknown etiology. In up to $25 \%$ it appears as a paraneoplastic syndrome. Here we discuss a 68-year old woman, who developed dermatomyositis one year after diagnosis of ovarial carcinoma. Initially, immunosuppressive therapy with prednisolone and the initiation of chemotherapy led to a sufficient control of the disease. Within the following months she developed cutaneous erosions, which were interpreted as adverse events of cytostatics and repeatedly led to change or cessation of chemotherapy. At this point of time the ovarial carcinoma appeared progredient. However, after continuation of chemotherapy all cutaneous erosions disappeared. This case demonstrates an improved therapy outcome of paraneoplastic dermatomyositis, which can be achieved by interdisciplinary co-operation of oncologists and dermatologists. Moreover, this case underlines the hypothesis, that tumorprogression is associated with exacerbation of dermatomyositis and that oncologic therapy is essential for control of paraneoplastic dermatomyositis.

\section{Literatur}

1 Hengstman GJ, Brouwer R, Egberts WT, Seelig HP, Jongen PJ, van Venrooij WJ, van Engelen $B G$. Clinical and serological characteristics of 125 Dutch myositis patients. Myositis specific autoantibodies aid in the differential diagnosis of the idiopathic inflammatory myopathies. J Neurol 2002; 249: 69-75

2 Hill CL, Zhang Y, Sigurgeirsson B, Pukkala E, Mellemkjaer L, Airio A, Evans SR, Felson DT. Frequency of specific cancer types in dermatomyositis and polymyositis: a population-based study. Lancet 2001; 357: $96-100$

3 Callen JP. Dermatomyositis. In: Hertl M (ed). Autoimmune Diseases of the Skin, 2nd edn. Wien, New York: Springer, 2005: 229-244

4 Levine SM. Cancer and myositis: new insights into an old association. Curr Opin Rheumatol 2006; 18: 620-624

5 Callen JP. The value of malignancy evaluation in patients with dermatomyositis. J Am Acad Dermatol 1982; 6: 253 - 259

6 Iorizzo LJ 3rd, Jorizzo JL. The treatment and prognosis of dermatomyositis: An updated review. J Am Acad Dermatol 2008; 59: 99-112

7 Kaufmann J, Hunzelmann N, Genth E, Krieg T. The clinical spectrum of dermatomyositis. J Dtsch Dermatol Ges 2005; 3: 181 - 194

8 Di Berardino C, Mascaretti G. Ovarian cancer after kidney transplantation. Eur J Gynaecol Oncol 2008; 29: 89-92

9 Schein PS, Winokur SH. Immunosuppressive and cytotoxic chemotherapy: long-term complications. Ann Intern Med 1975; 82: 84-95

10 Mori $\mathrm{H}$, Habe K, Hakamada A, Isoda K, Mizutani H. Relapse of dermatomyositis after 10 years in remission following curative surgical treatment of lung cancer. J Dermatol 2005; 32: 290-294

11 Osako T, Ito Y, Morimatsu A, Tada K, Sakurai N, Takahashi S, Akiyama F, Iwase T, Hatake K. Flare-up of dermatomyositis along with recurrence of breast cancer. Breast J 2007; 13: 200-202

12 Przybylski G, Jarzemska A, Czerniak J, Siemiatkowska K, Gadzinska A, Cieslinski K. A case report of a patient with dermatomyositis as a prodromal sign of lung cancer. Pol Arch Med Wewn 2008; 118: 143-147

13 Takahashi F, Tsuta K, Nagaoka T, Miyamoto H, Saito Y, Amano H, Uchida K, Morio Y, Shimizu K, Sasaki S, Tominaga S, Uekusa T, Izumi H, Anami Y, Matsuno Y, Takahashi K, Fukuchi Y. Successful resection of dermatomyositis associated with thymic carcinoma: report of a case. Surg Today 2008; 38: $245-248$

14 Zang YS, Xiu QY, Fang Z, Li B, Xia TB. Case report: dramatic recovery of lung adenocarcinoma-associated dermatomyositis with targeted lung cancer therapy alone. Oncologist 2008; 13: 79-81 